\title{
Age-Related Changes in the Oligodendrocyte Progenitor Pool Influence Brain Remodeling after Injury
}

\author{
Jamie Wright ${ }^{a, b}$ Gui Zhang ${ }^{a, b}$ Tzong-Shiue Yu ${ }^{a, b}$ Steven G. Kernie ${ }^{a, b}$ \\ Departments of a Pediatrics and ${ }^{b}$ Developmental Biology, University of Texas Southwestern Medical Center, \\ Dallas, Tex., USA
}

\section{Key Words}

Neural stem cell · White matter · Oligodendrocyte progenitor cells · Traumatic brain injury · Platelet-derived growth factor receptor- $\alpha$

\begin{abstract}
Brain remodeling occurs after all forms of brain injury, though the mechanisms underlying this phenomenon are mostly unknown. Neural stem and progenitor cells are one source of endogenous cells that may contribute to brain remodeling and subsequent recovery. In addition, certain populations of progenitors are particularly susceptible to injury, and their depletion may lead to the impairment of developmental processes that vary with age. We particularly focus on glial progenitors, which are more abundant postnatally and particularly susceptible to acquired brain injuries such as traumatic brain injury. We have recently characterized a novel transgenic mouse that expresses herpes thymidine kinase under the control of the neural-progenitor-specific nestin promoter and allows for temporally induced ablation of dividing progenitors. By genetically depleting dividing cortical progenitors at various times, we identify postnatal day 7 (P7) to P14 as a critical period for oligodendrogenesis. Targeted ablation of dividing progenitors dur-
\end{abstract}

\section{KARGER}

Fax +4161306 1234

E-Mail karger@karger.ch

www.karger.com (c) 2010 S. Karger AG, Basel

Accessible online at: www.karger.com/dne ing this window leads to cell-specific depletion of oligodendrocyte precursors expressing platelet-derived growth factor receptor- $\alpha$ and corresponding myelination and motor deficits. This modeling provides insight into how the age at which white matter injury occurs influences both injury severity and subsequent recovery.

Copyright $\odot 2010$ S. Karger AG, Basel

\section{Introduction}

With traumatic brain injury (TBI) as the leading cause of acquired brain injury in both children and adults, elucidating how damage occurs, the brain's response to that injury, and how both might be affected in order to obtain the best possible outcome are of significant clinical relevance [Giza and Prins, 2006; Yu et al., 2008; Giza et al., 2009; Sookplung and Vavilala, 2009]. The study of pediatric brain injury poses unique challenges because the sequelae of these injuries are age dependent. Similar injuries can have drastically different effects depending on the age at which they occur [Back et al., 2001; Giza and Prins, 2006; Giza et al., 2009; Sookplung and Vavilala, 2009]. In the past decade, neurogenesis in the adult central nervous system (CNS) has become a widely accepted 
phenomenon, and is now the subject of intense research for its therapeutic potential [Kernie and Parent, 2010]. In mammals, populations of neural stem cells (NSC) in the dentate gyrus of the hippocampus and the subventricular zone of the lateral ventricles are known to proliferate and differentiate throughout the lifespan [Altman and Das, 1965; Luskin, 1993; Gage et al., 1995; Suhonen et al., 1996; Kernie and Parent, 2010]. Insults such as hypoxia-ischemia and TBI are known to affect these NSC populations in a maturation-dependent manner [Back et al., 2001; Jin et al., 2001; Miles and Kernie, 2008; Yu et al., 2008]. In order to study these injuries with reasonable specificity, it is critical to know the timing at which various progenitors populate and mature throughout the brain, and the effects of insults to these populations on development. For additional information on this topic see also the article by Covey et al. [2010] in this issue of Developmental Neuroscience.

The major cell types of the mammalian CNS arise in three temporally distinct, although overlapping, waves [Sauvageot and Stiles, 2002]. Neurogenesis occurs first, peaking around embryonic day (E) 14 in mice, followed by gliogenesis, which peaks between postnatal days $(\mathrm{P}) 0$ and 2 , and then finally, oligodendrogenesis occurs, peaking about P14 [Sauvageot and Stiles, 2002]. These same progenitor pools follow a similar path in humans, though the exact ages at which these changes occur are not as well known. Much of the age-specific effects associated with TBI and other acquired brain diseases are likely secondary to these discrete developmental stages of progenitor proliferation. By using a neural progenitor-specific promoter to drive the expression of enhanced green fluorescent protein (eGFP), we have been able to utilize these temporally distinct peaks in cellular genesis to target specific progenitor cell populations for the investigation of age-related changes in the progenitor pool [Koch et al., 2008; Yu et al., 2008; Kernie and Parent, 2010].

While significant progress has been made in our understanding of neuronal progenitors, much less is known in regard to glial progenitors, despite the fact that white matter is crucial to normal brain function. Periventricular white matter injury is the major form of brain injury and the leading cause of neurological disability such as motor impairment (i.e. cerebral palsy) in survivors of premature birth [Back et al., 2002; Volpe, 2003; Back et al., 2007; Segovia et al., 2008]. Periventricular white matter injury encompasses a spectrum of white matter injuries including both focal cystic necrotic lesions, known as periventricular leukomalacia, and diffuse myelination disturbances [Back et al., 2007]. White matter changes also frequently accompany TBI and contribute to much of the morbidity associated with severe injury [Tasker et al., 2005; Tasker, 2006; Sookplung and Vavilala, 2009; Gale and Prigatano, 2010].

Based on various published studies, age-dependent vulnerability in the progenitor pool is already apparent [Back et al., 2002; Koch et al., 2008; Miles and Kernie, 2008; Segovia et al., 2008]. In this study, we expand upon what is already known by characterizing age-related changes in the oligodendrocyte progenitor populations. We then show how a recently generated transgenic animal expressing herpes simplex virus thymidine kinase (HSV-TK) under the control of the nestin promoter can be used in the study of age-dependent vulnerability of the progenitor pool, and how this may apply to recovery following traumatic and other forms of brain injury.

\section{Materials and Methods}

\section{Animals}

All mice used in these experiments were housed and cared for in the UT Southwestern Medical Center's Animal Resource Center, which is certified by the Association for Assessment and Accreditation of Laboratory Animal Care. All animal protocols received prior approval by the institutional animal use and care committee at the UT Southwestern Medical Center in accordance with the highest standards for the humane use of animals in biomedical research.

\section{Ganciclovir Administration}

For P14 animals, ganciclovir (200 mg/kg/day; Cytovene-IV; Roche Pharmaceuticals, San Francisco, Calif., USA) or vehicle (distilled $\mathrm{H}_{2} \mathrm{O}$ ) was delivered via microosmotic pump at a constant infusion rate of $0.25 \mu \mathrm{l} / \mathrm{h}$ for 2 weeks (model 1002; Alzet, Cupertino, Calif., USA). Pumps were implanted per the Alzet protocol as described below. Two-week-old mice were anesthetized with isoflurane and a small incision was made between the scapulae. A hemostat was used to spread the subcutaneous connective tissue to form a small pocket, and the pump was inserted into this pocket with the flow moderator pointing away from the incision. The skin incision was closed with sutures. For treatment of P7 animals, ganciclovir $(100 \mathrm{mg} / \mathrm{kg} / \mathrm{day})$ was delivered via daily intraperitoneal injections for 1 week. For in utero treatment, ganciclo$\operatorname{vir}(200 \mathrm{mg} / \mathrm{kg} / \mathrm{day})$ was administered once daily via intraperitoneal injection to pregnant transgenic animals from E15.5 to E19.5.

\section{Immunohistochemistry}

Twenty-four hours after treatment, the mice were deeply anesthetized with a ketamine/xylazine mixture prior to transcardiac perfusion with phosphate-buffered saline (PBS) followed by $4 \%$ paraformaldehyde for $10 \mathrm{~min}$ each. Their brains were dissected out and fixed overnight in $4 \%$ paraformaldehyde. The following day, the brains were embedded in $3 \%$ agarose and serial $50-\mu \mathrm{m}$ coronal sections encompassing the entire hippocampus were cut using a vibrating microtome (VT1000S; Leica Microsystems, 
Bannockburn, Ill., USA). The sections were collected and every sixth section was used for immunohistochemistry. Free-floating sections were mounted onto glass slides, then dried thoroughly prior to staining. The sections were washed 3 times in $0.3 \%$ Triton $\mathrm{X}-100$ in PBS, and then blocked with 5\% normal donkey serum (NDS) in $0.3 \%$ Triton X-100 at room temperature for $1 \mathrm{~h}$. Following initial blocking, the slides were washed in PBS, then blocked with Fc Receptor Block (Innovex Biosciences, Richmond, Calif., USA) for $30 \mathrm{~min}$ at room temperature followed by an additional short PBS wash. All primary antibodies were combined into a total volume of $500 \mu \mathrm{l}$ of $2.5 \%$ NDS in $0.3 \%$ Triton X-100 PBS at the appropriate concentrations and incubated at $4{ }^{\circ} \mathrm{C}$ overnight. The sections were then washed 3 times in $0.3 \%$ Triton X-100 and then incubated with the secondary antibodies diluted to the appropriate concentrations in $2.5 \%$ NDS in $0.3 \%$ Triton X-100 PBS for $1.5 \mathrm{~h}$ at room temperature. The sections were then washed 3 times in $0.3 \%$ Triton X-100 PBS and once in PBS. The slides were then dried and covered with plastic cover slips using Immu-Mount (Thermo Scientific, Waltham, Mass., USA).

Primary antibodies used were mouse anti-myelin basic protein (anti-MBP; 1:500; Covance, Princeton, N.J., USA), rat antiplatelet-derived growth factor receptor- $\alpha$ (anti-PDGFR- $\alpha$; 1:250; BD Pharmingen, Pasig City, Republic of the Philippines) and rabbit anti-Ki-67 (1:200; Thermo Scientific). All secondary fluorescent antibodies were raised in donkey serum and used at a concentration of 1:200 (Jackson Laboratories, Bar Harbor, Me., USA).

Staining for stereological counting was done on $50-\mu \mathrm{m} \mathrm{sec-}$ tions collected as described above. Free-floating sections were washed, then quenched in $0.3 \%$ hydrogen peroxide, washed and then blocked in $5 \%$ normal goat serum in $0.3 \%$ Triton X-100 PBS at $4{ }^{\circ} \mathrm{C}$ overnight to reduce background. The sections were then incubated in primary antibody at $4^{\circ} \mathrm{C}$ overnight to maximize antibody penetration, followed by incubation in biotinylated secondary antibody. The sections were then incubated in horseradish-peroxidase-based Vectastain ABC Kit (Vector Laboratories, Burlingame, Calif., USA), followed by incubation in Vector NovaRED Substrate Kit according to the manufacturer's protocol for amplification and visualization of staining (Vector Laboratories). Primary antibodies used included rabbit anti-PDGFR- $\alpha$ (1:250; Santa Cruz Biotechnology, Santa Cruz, Calif., USA) and mouse anti-MBP (1:250; Covance, Princeton, N.J., USA). Secondary antibodies were used at a concentration of 1:500 and purchased from Vector Laboratories.

\section{Microscopy}

Fluorescent staining was photographed using Zeiss (Thornwood, N.Y., USA) LSM 510 scanning confocal multicolor microscopy using argon 488, He 543 and He 633 lasers (version 3.2 SP2, Aim interface) and a Zeiss Neofluar $25 \times / 0.81-\mathrm{mm}$ oil DIC lens. Stereological quantification was performed on DAB-stained sections using an Olympus BX51 System Microscope with a MicroFIRE A/R camera (Optronics, Muskogee, Okla., USA). Cell quantification was conducted using the Optical Fractionator Probe within the Stereo Investigator software (MBF Bioscience, MicroBrightField Inc., Williston, Vt., USA). Counting was done using a $\times 40$ lens. A 500 by $500 \mu \mathrm{m}$ area of cortex $200 \mu \mathrm{m}$ lateral to the midline and $200 \mu \mathrm{m}$ above the corpus callosum was counted. Seven sections were counted for each animal, with an average of 210 counting fields per animal and an average section thickness following processing of approximately $35 \mu \mathrm{m}$.

Brain Progenitor Pool after Injury
In order to reduce bias between samples, all tissue was processed in the same manner (as described in the Immunohistochemistry subsection). In order to minimize the effect of shrinkage, a height sampling fraction of $30 \mu \mathrm{m}$ was used to account for actual postprocessing thickness. Due to the nature of the counting region, an area sampling fraction of 1 was used. A section sampling fraction of 6 was used (every sixth section was counted). Only slides with homogenous staining and all sections present were counted, and the coefficient of variation for each animal quantified was less than $15 \%$.

\section{Western Blotting}

Animals were sacrificed $24 \mathrm{~h}$ following the treatment period, and hippocampus and cortex tissue samples were collected. Samples were homogenized and cellular debris was removed by centrifugation for PDGFR- $\alpha$ and MBP immunoblotting. Protein concentrations were determined for each sample using a BCA Protein Assay (Thermo Scientific). For MBP immunoblotting, 10 $\mu \mathrm{g}$ of protein was used per well, and $20 \mu \mathrm{g}$ of protein was used per well for PDGFR- $\alpha$ blotting. A $10 \%$ polyacrylamide gel was used for PDGFR- $\alpha$ blotting, while a 15\% gel was used for MBP immunoblotting.

After the gels were run, protein was transferred to a polyvinylidene fluoride membrane and blocked for $1 \mathrm{~h}$ in $5 \%$ nonfat milk at room temperature. The membrane was incubated in primary antibody for $2 \mathrm{~h}$ at room temperature, washed, and then incubated in secondary antibody for $1 \mathrm{~h}$ at room temperature. Primary antibodies used included mouse anti-MBP (1:1,000; Covance), rabbit anti-PDGFR- $\alpha$ (1:1,000; Santa Cruz Biotechnology) and mouse anti- $\beta$-tubulin (1:30,000; Sigma-Aldrich, St. Louis, Mo., USA). All secondary antibodies were raised in goat serum and used at a concentration of 1:10,000 (Vector Laboratories). All washes were conducted in PBS with $0.2 \%$ Tween. Protein bands were visualized using Lumi Light Western Blotting Substrate (Roche, Basel, Switzerland).

\section{Wire Hang}

P7 HSV-TK-positive $(\mathrm{n}=6)$ and HSV-TK-negative $(\mathrm{n}=6)$ mice were given ganciclovir (100 mg/kg/day) via intraperitoneal injection for 7 days. The animals survived and underwent wire hang testing (Letica, Bioseb, France) on P15 to assess gross motor strength. The mice were suspended by their forepaws on a thin metal wire over a cushioned box, and latency to fall was recorded. Each animal underwent 3 trials 10 min apart.

\section{Statistical Analysis}

Statistical analysis was performed with Prism 5 software. The t test (for paired data) or one-way ANOVA (for nonparametric data) with Bonferroni post hoc correction was used for data analysis.

\section{Result}

\section{Glial Progenitors Peak prior to Myelination, Which} Occurs after Neurogenesis Is More Quiescent

The neuronal progenitor population has been the focus of extensive research from which significant progress 
has been made in the understanding of both its normal development and its response to injury [Parent et al., 2002; Emsley et al., 2005; Richardson et al., 2007; Miles and Kernie, 2008; Yu et al., 2008; Kernie and Parent, 2010]. Less is known regarding the glial progenitor population. As is the case with neuronal progenitors, there are different types of glial progenitors, which are distinguishable from each other by the proteins they express. The earliest form of oligodendrocyte progenitors expresses PDGFR- $\alpha$, whereas mature oligodendrocytes express myelin-associated markers such as MBP [Woodruff et al., 2001; Back et al., 2007]. Although there are some data regarding these progenitors in the developing rat, the degree of proliferation of this glial progenitor population has not been as well studied in mice [Pringle et al., 1992; He et al., 2009].

In order to gain a more thorough understanding of the timeline of oligodendrocyte progenitor development in mice, we established profiles using the progenitor marker PDGFR- $\alpha$ and the mature oligodendrocytic marker MBP. We obtained sections encompassing the hippocampus and corpus callosum of wild-type mice at P0, P7, P14, P21, P28 and P60, and performed immunohistochemistry and Western blot analysis (fig. 1). A steady decline in PDGFR- $\alpha$ expression is apparent over the period from P0 to P60 with minimal expression after P21 (fig. 1a-f, $\mathrm{m})$. We also observed a corresponding increase in MBP expression from P0 to $\mathrm{P} 60$ with no significant expression appearing until P21 (fig. 1g-l, n). These trends in PDGFR- $\alpha$ and MBP expression correspond with the onset of myelination at P21, which follows the decline in neuronal progenitor proliferation [Sauvageot and Stiles, 2002; Koch et al., 2008]. We also analyzed the presence of PDGFR- $\alpha$ and MBP in the cortex along with the proliferation marker $\mathrm{Ki}-67$, which is specific for all phases of cycling cells but is absent from resting cells [Scholzen and Gerdes, 2000]. Here, we observed many proliferating PDGFR- $\alpha$-expressing progenitors at early time points, P0 and P7, but fewer at later times. Interestingly, however, the proliferation of cortical PDGFR- $\alpha$-expressing cells persisted through P60 (fig. 1o-t, arrows).

\section{Ablation of Early Neural Progenitors Causes Profound Cortical Dysplasia}

We recently developed a novel transgenic mouse line that contains a modified version of the HSV-TK in addition to eGFP, downstream from the nestin promoter and its second intron regulatory element [Yu et al., 2008]. This nestin-HSV-TK transgenic mouse line allows for the specific, controlled ablation of dividing type 1 and 2a neural progenitors by administering the antiviral drug ganciclovir [Yu et al., 2008]. Using this mouse line, we are able to selectively ablate dividing neural progenitors at any point in development.

While many of the current studies focus on the perinatal period, studies looking at prenatal time points shed light on early development and the pathophysiology of certain developmental disturbances [Takashima et al., 2009]. We first administered ganciclovir by injection once daily to pregnant transgenic mice at E15.5-E19.5, during the peak of cortical neurogenesis. We observed that at $\mathrm{P} 0$, the entire forebrain of transgenic pups exposed to ganciclovir was ablated, whereas the already formed hindbrain was mostly intact (fig. 2a-c). This demonstrates that, prenatally, when there is a relatively nonspecific abundance of dividing progenitors, injuries to this population result in very profound cortical deficits.

\section{Ablation of Postnatal Progenitors Leads to Depletion of Cortical Oligodendrocyte Precursors at Early \\ Postnatal Stages}

Since white matter injury is so common in infants and young children after a variety of brain injuries including TBI, we investigated whether the age at which the injury occurs might affect cortical myelination. We first took nestin-HSV-TK transgenic animals and treated them from either P7-P14 or P14-P28 with ganciclovir to mimic roughly the newborn and infant (P7-P14) and the young toddler to early school-aged (P14-P28) equivalents of human white matter development. Here we find that early treatment from P7 to P14 essentially eliminates PDGFR- $\alpha$-expressing glial progenitors from the cortex. This is demonstrated using histology with stereologybased cell counting as well as Western blot analysis for overall quantification (fig. 3a-d). Later treatment from P14 to P28, however, resulted in no significant differences in the cortical progenitor population either by cell counts or Western blot (fig. 3e-h). These data suggest that at the time when progenitors are dividing and migrating and are known to be vulnerable to injury, their presence is required at this critical window in order for the normal cortical population to be present.

We next examined whether elimination of dividing progenitors affected cortical myelination at these time points. By performing triple immunostaining with Ki-67, PDGFR- $\alpha$ and MBP, we were able to demonstrate that the dividing Ki-67 population is essentially entirely eliminated following exposure to ganciclovir from P7 to P14, but when exposed from P14 to P28, there is essentially no change since the proliferating population is so much low- 

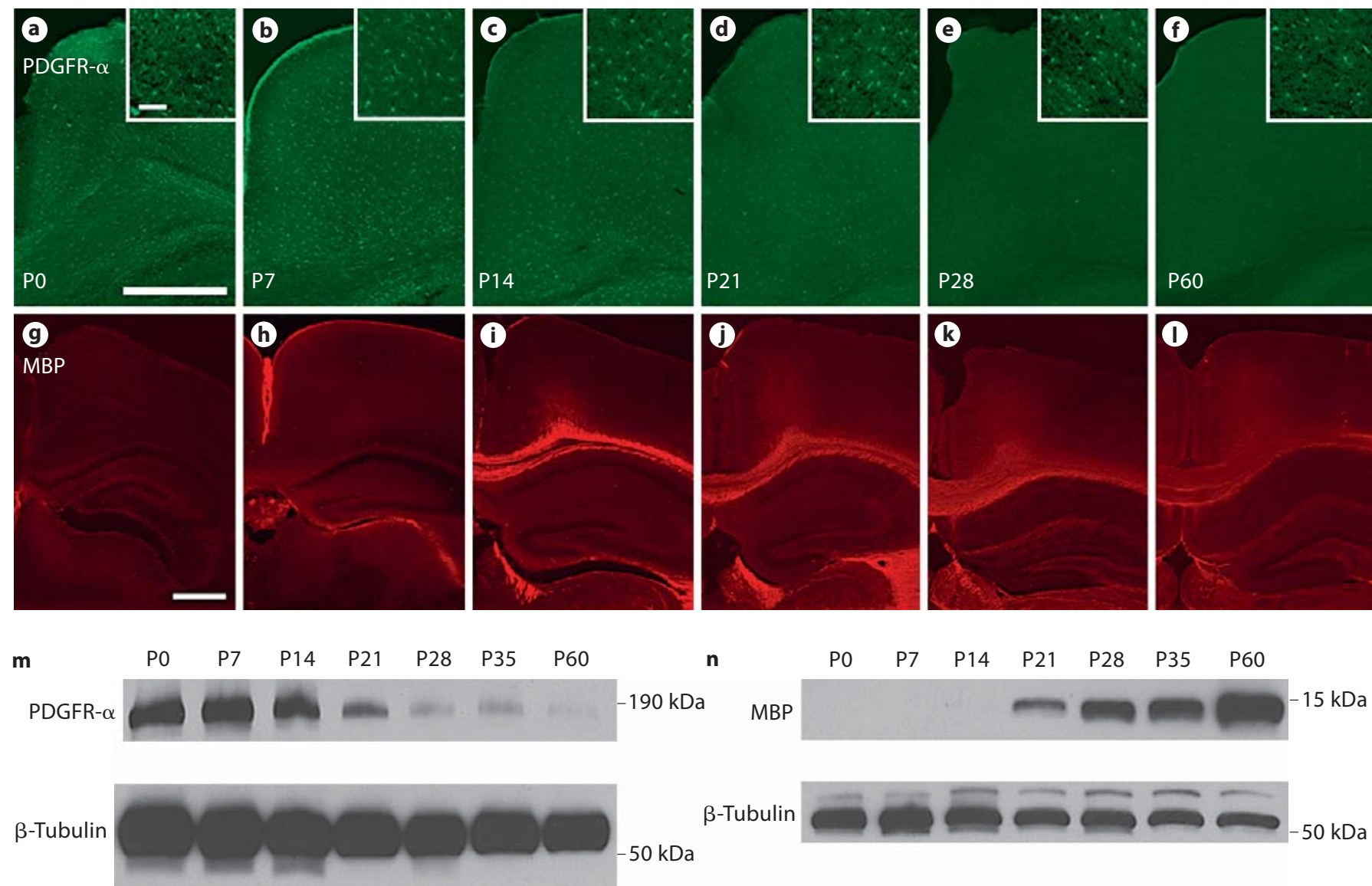

Ki67 PDGFR- $\alpha$ MBP
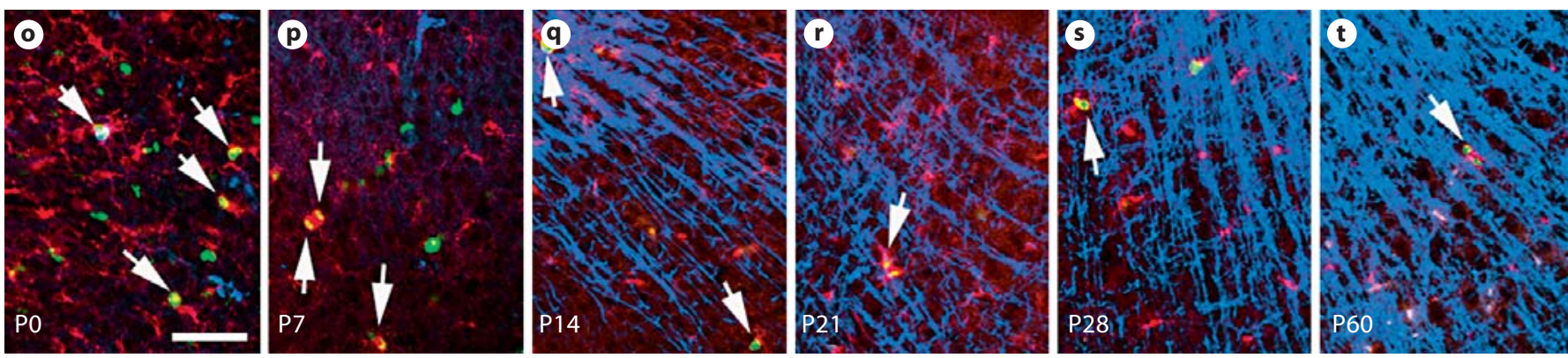

Fig. 1. During normal development, the PDGFR- $\alpha$-expressing oligodendrocyte progenitor population declines as the number of MBP-expressing mature oligodendrocytes increases. Cortical sections taken from wild-type animals at P0-P60 were stained for the oligodendrocyte progenitor marker PDGFR- $\alpha(\mathbf{a}-\mathbf{f})$ and the mature oligodendrocyte marker MBP (g-I) using immunohistochemistry. Higher-magnification images of sections taken over the period from P0 to P60 stained with PDGFR- $\alpha$, MBP and the

proliferation marker Ki-67 demonstrate that proliferating oligodendrocyte progenitors diminish over time as myelination occurs, though proliferative PDGFR- $\alpha$-expressing progenitors persist at least through 2 months of age (o-t, arrows). Scale bars $=500$ $\mu \mathrm{m}(\mathbf{a}, \mathbf{g})$ and $50 \mu \mathrm{m}(\mathbf{a}$, inset, o). $\mathbf{m}, \mathbf{n}$ Western blot analysis of cortical samples taken from animals ranging in age from P0 to P60 was used to characterize PDGFR- $\alpha(180 \mathrm{kDa} ; \mathbf{m})$ and MBP (14 kDa; $\mathbf{n})$ expression. 

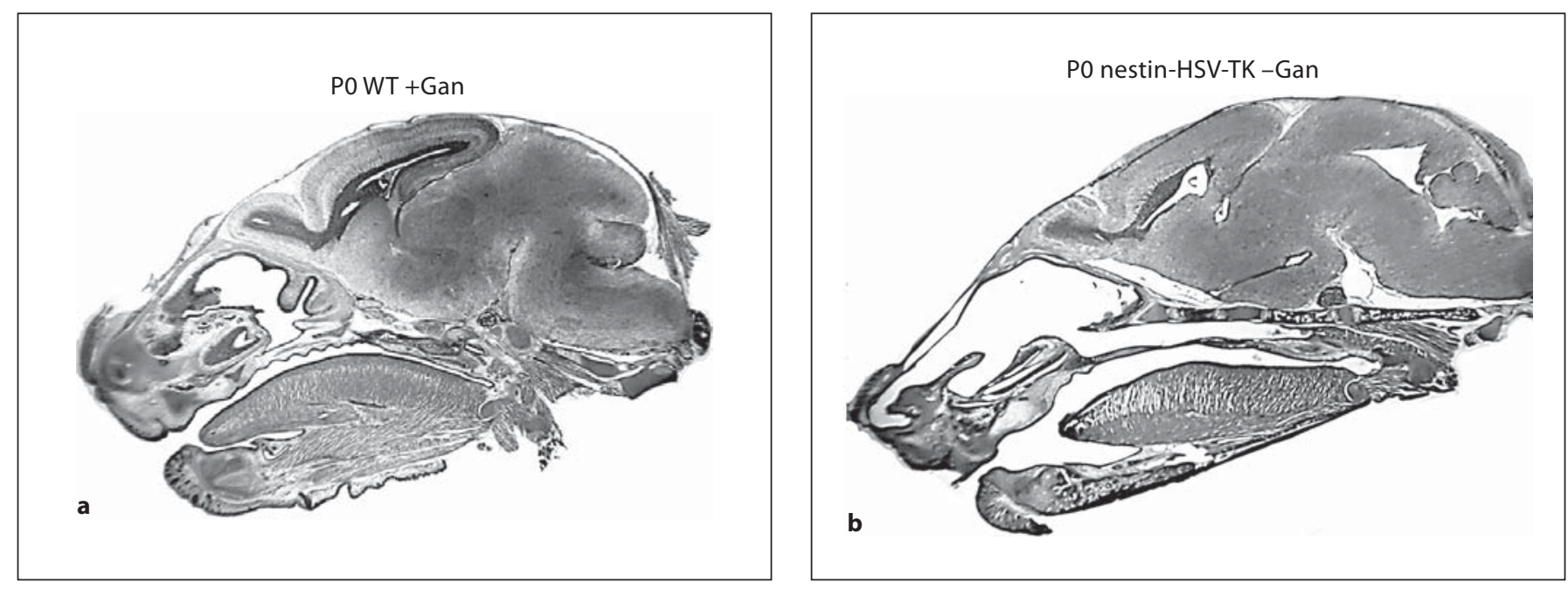

Fig. 2. Prenatal ablation of dividing neural progenitors impairs cortical development. Pregnant nestin-HSV-TK mice were treated with ganciclovir (+Gan) daily (200 mg/kg/day) via intraperitoneal injection from E15.5 to E19.5. Sections were taken at P0 (a-c). The forebrain of treated transgenic animals is completely ablated (c; arrows), while the hindbrain, which has been formed prior to treatment, remains intact (c).

er during the later developmental window (fig. 4). MBP staining overall does not look qualitatively much different in this section of the cortex for either group (fig. 4).

\section{Ablation of Postnatal Progenitors Leads to}

\section{Myelination and Motor Deficits at Early Postnatal}

\section{Stages}

Although the absence of cortical progenitors following ganciclovir-induced ablation of dividing progenitors suggests subsequent myelination problems, we tested this by performing Western blot analysis on early- and late-treated transgenic mice with MBP. Here, we observed that mice treated from P7 to P14 demonstrated loss of overall hippocampal MBP by Western blot (fig. 5a). This was not observed in later-treated animals from P14 to P28 (not shown). In addition, we performed a wire hang motor be-

havior assay on both groups of animals following ganciclovir treatment. Only those treated from P7 to P14 showed persistent motor deficits when compared to the control population (fig. 5b).

\section{Discussion}

Age-related differences in recovery following brain injury have long been observed in rodent and primate models, and more recently in humans [Giza et al., 2009]. Neural development continues throughout childhood and adolescence, with waves of synaptogenesis followed by subsequent pruning taking place in each of the major cortical regions, starting in the occipital lobe in the early postnatal period and concluding in the frontal lobe dur- 


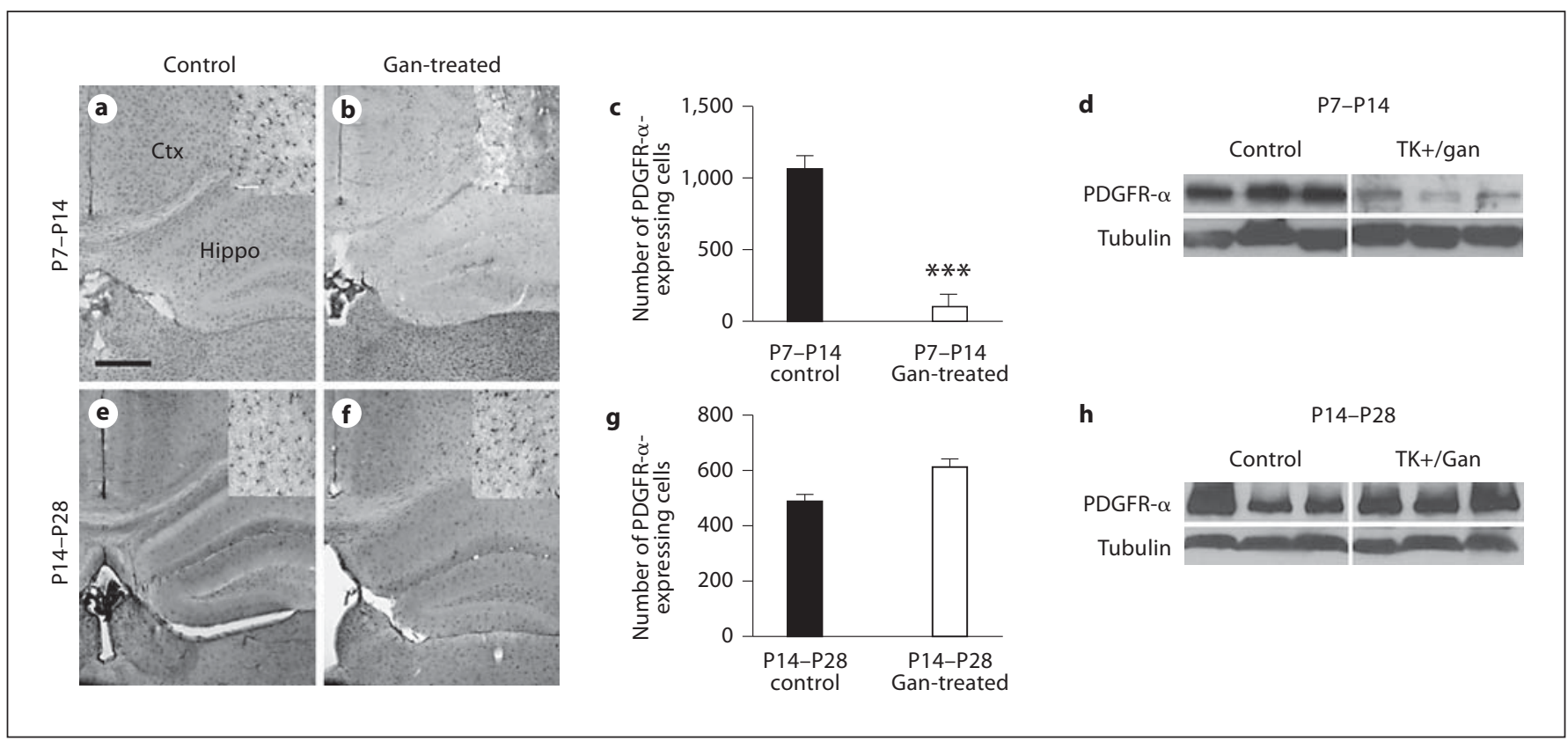

Fig. 3. Ganciclovir (Gan) treatment in nestin-HSV-TK transgenic mice depletes PDGFR- $\alpha$-expressing glial progenitors in the cortex when given from P7 to P14. Nestin-HSV-TK mice were given Gan daily from P7 to P14. Examination of the cortex at P15 revealed that the PDGFR- $\alpha$-expressing population was almost entirely depleted $(\mathbf{a}, \mathbf{b})$. Stereological counts were performed on a subset of cortex, and there was an over 10-fold decrease in cell number in animals treated with Gan (c). Similarly, Western blot analysis at P15 shows depletion of PDGFR- $\alpha$ from the entire cortex (d). In contrast, animals treated with Gan from P14 to P28 show no difference in PDGFR- $\alpha$ cell number or Western blot quantification when analyzed at P29 (e-h). Insets in $\mathbf{a}, \mathbf{b}, \mathbf{e}$ and $\mathbf{f}$ are higher-magnification images of representative areas counted in $\mathbf{c}$ and $\mathbf{g}$. For $\mathbf{c}$ and $\mathbf{g}: \mathrm{n}=4$ controls and 5 treated for each group. ${ }^{* * *} \mathrm{p}<0.0001$. Scale bars $=500 \mu \mathrm{m}$ (a) and $50 \mu \mathrm{m}$ (a, inset). ing late adolescence and early adulthood [Giza et al., 2009; Johnston, 2009]. This ongoing development is believed to be why pediatric patients appear to have an advantage over adult patients in some aspects of recovery following TBI [Giza et al., 2009; Johnston, 2009]. Contrary to this 'younger is better' paradigm, follow-up studies have shown that even mild TBI often has long-term behavioral effects, some of which appear to 'come out' with age as patients reach developmental milestones not achieved prior to injury [Giza et al., 2009; Goold and Vane, 2009; Sookplung and Vavilala, 2009]. The diverse neurological and cognitive effects of TBI have been found to have a particularly profound impact on hippocampal and forebrain function [Giza et al., 2009; Kernie and Parent, 2010]. This, in addition to the self-recovery observed in all but the most severe cases, suggests that forebrain neurogenesis may play an important role in recovery following TBI [Richardson et al., 2007; Kernie and Parent, 2010].

The response of NSC to TBI has been characterized using a transgenic mouse line containing eGFP under the control of the neural stem- and progenitor cell-specific nestin promoter and its second intron regulatory element [Yu et al., 2008]. This nestin-eGFP mouse line allows for making distinctions between the different cell types in order to identify vulnerable populations. Using this nestin-eGFP mouse in a well-established model for TBI, controlled cortical impact, type 2 DCX-expressing cells were found to have heightened vulnerability to injury, while the type 1 earlier eGFP-expressing progenitors, which are normally more quiescent, are induced to proliferate following controlled cortical impact [Yu et al., 2008]. Studies like this highlight how the same injury can affect adjacent cell populations quite differently depending on how far along the maturation curve they have progressed. This has significant implications on how one might recover from the same acquired brain injury depending on how many progenitors are present in the injured area and at what age the injury occurs.

Less is known about the oligodendrocyte progenitor cell population following TBI, even though changes in white matter are some of the most apparent after injury. 


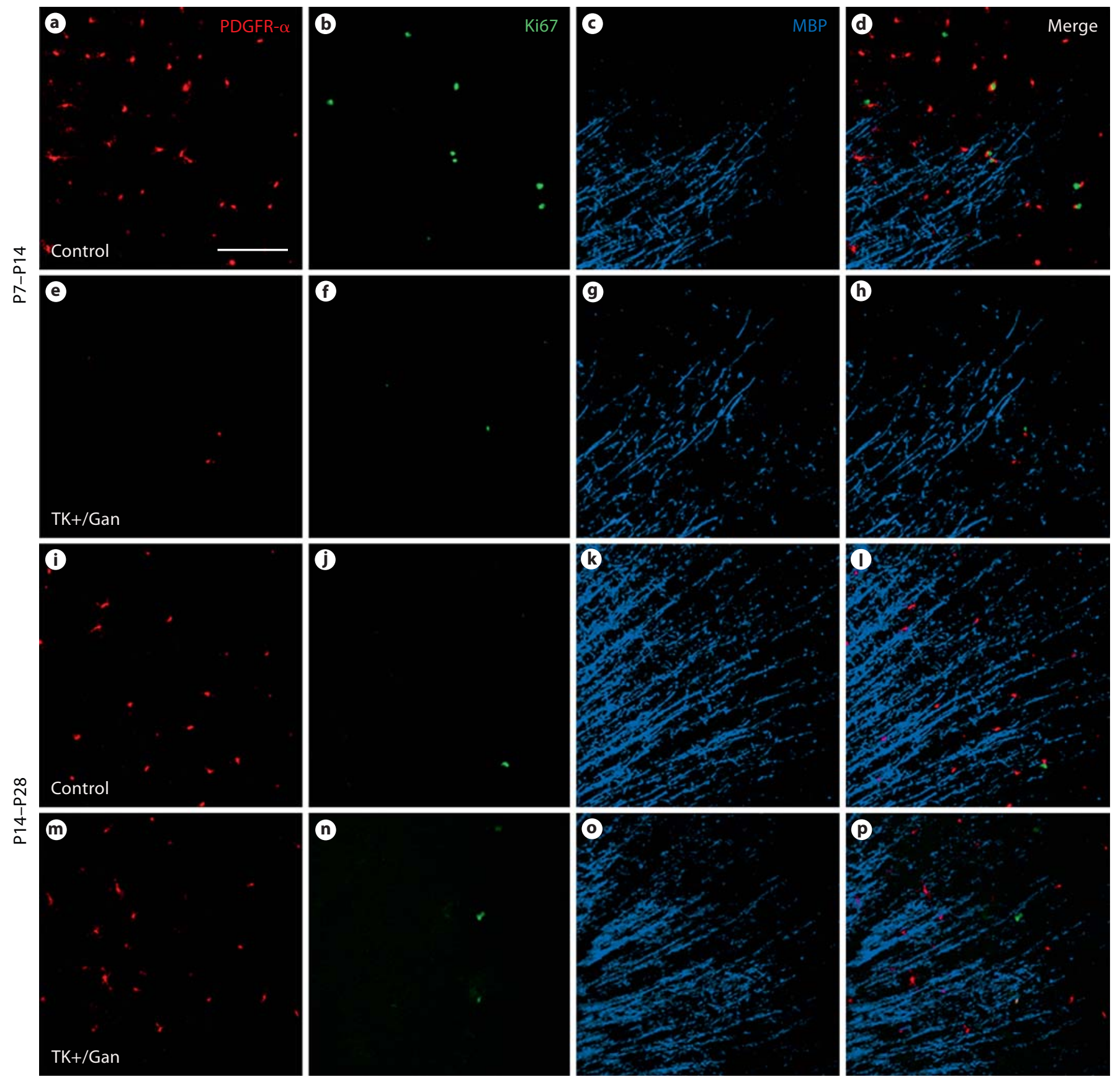

Fig. 4. Dividing progenitors from nestin-HSV-TK transgenic mice are depleted when treated from P7 to P14. The pan-dividing cell marker Ki-67 was used in conjunction with PDGFR- $\alpha$ and MBP, and only in the animals treated from P7 to P14 were Ki-67- expressing dividing cells depleted (a-h). In animals treated from P14 to P28, there were fewer Ki-67-expressing cells in both the control and treated groups, with no differences noted between the two (i-p). Gan = Ganciclovir. Scale bar $(\mathbf{a})=100 \mu \mathrm{m}$. 
Fig. 5. Nestin-HSV-TK mice treated from P7 to P14 have impaired myelination and motor function. Western blot analysis was performed on hippocampal tissue from P15 animals for MBP, which was highly diminished in animals treated from P7 to P14 (a). At P15, animals were tested for motor function using a wire hang device, and those treated from P7 to P14 were impaired compared to controls (b; $\mathrm{n}=5$ for each group). ${ }^{*} \mathrm{p}<0.05$. Gan $=$ Ganciclovir.

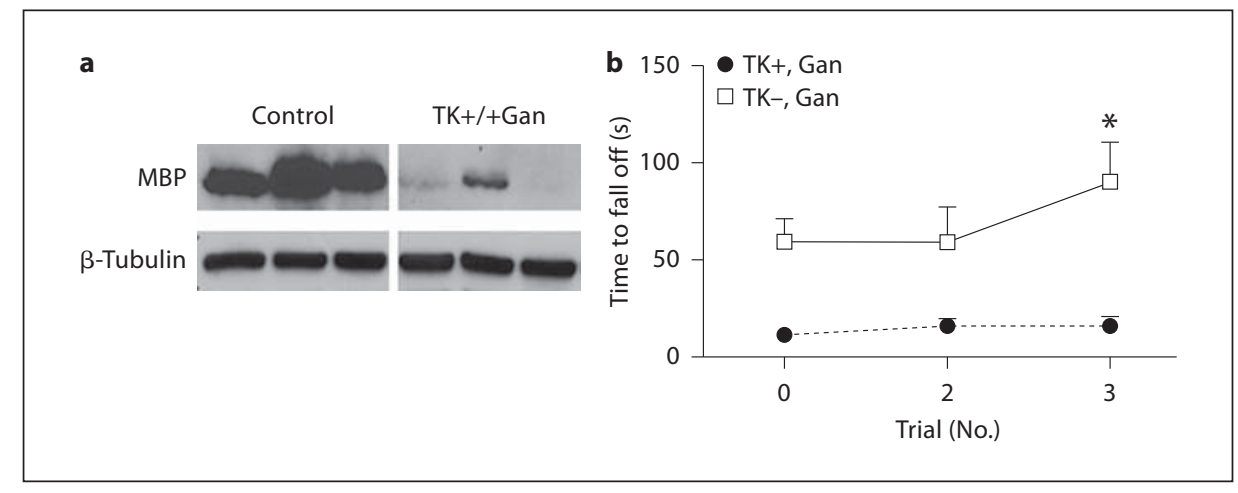

Work done previously using a hypoxic-ischemic neonatal rat model demonstrated that the mechanism of white matter injury, like neuronal injury, involves maturationdependent vulnerability [Back et al., 2002, 2007; Segovia et al., 2008]. As in the neuronal progenitor population, there are different types of oligodendrocytes depending on their level of differentiation, which are distinguishable from each other by the proteins they express and their capacity to proliferate. The earliest form of oligodendrocytes is the progenitor which gives rise to the preoligodendrocyte population [Back et al., 2007]. These preoligodendrocytes then give rise to postmitotic, immature oligodendrocytes, which then finally differentiate into mature oligodendrocytes [Back et al., 2007]. Preoligodendrocytes and late progenitors have been found to be more susceptible to glutamate-induced cell death and oxidative stress following hypoxia-ischemia than mature oligodendrocytes [Back et al., 2002; Ness et al., 2004; Back et al., 2007; Segovia et al., 2008]. In mice, the peak of oligodendrogenesis occurs at 2 weeks postnatally, with the majority of myelination occurring between 3 and 6 weeks [Sauvageot and Stiles, 2002; Koch et al., 2008]. The period of vulnerability for periventricular white matter injury coincides with the peak of oligodendrogenesis, with a marked decline in incidence with the onset of maturation of the vulnerable preoligodendrocyte population [Back et al., 2001].

In addition to these effects on the white matter oligodendrocyte population, more recent findings suggest that insults also have significant effects on gray matter oligodendrocyte progenitors in the neocortex [Rothstein and Levison, 2005]. These gray matter oligodendrocytes, or satellite oligodendrocytes, are nonmyelinating cells believed to perform important homeostatic functions to sustain the neuronal microenvironment [Baumann and Pham-Dinh, 2001; Rothstein and Levison, 2005]. Recent studies have coined the term 'polydendrocytes' to describe these highly branched NG2 and PDGFR- $\alpha$-expressing oligodendrocyte progenitor cells, suggesting them as a fourth unique glial cell type [Nishiyama et al., 2009]. While they are known to differentiate into myelinating oligodendrocytes, they are now believed to have the potential to give rise to neurons and astrocytes as well and may even be integrated into the synaptic network [Nishiyama et al., 2009]. These findings suggest the diverse effects of insults to the oligodendrocyte progenitor cell population for brain homeostasis beyond those functions attributed to myelination.

Transgenic mouse models have emerged as an invaluable tool in biomedical research and have transformed the study of brain injury and its effects on the progenitor populations in the CNS. Such tools have now gone beyond examining the effects of merely ubiquitously deleting a gene from a given animal line, but now allow for more precise control of genetic perturbations. This is particularly valuable in the study of acquired brain injury such as TBI, where brain development is generally normal, but after the injury itself, a variety of cell-specific changes occur that can now be much more accurately assessed than what could be analyzed in the recent past using either nontransgenic or classical gene targeting strategies [Richardson et al., 2007; Kernie and Parent, 2010]. These models are particularly valuable in the characterization of the response of specific cell populations to stimuli because of their specificity and their ability to be temporally regulated. For example, prior to the use of genetically targeted cell ablation, the only way to model interruptions in development and acquired brain injury specific to the progenitor population was by irradiation or the use of chemical inhibitors, both of which were markedly nonspecific and resulted in secondary damage, making it difficult to determine causal relationships 
[Shors et al., 2001; Gourevitch et al., 2004; Rola et al., 2004; Leuner et al., 2006; Winocur et al., 2006; Wojtowicz, 2006; Yu et al., 2008]. Here, we have demonstrated how a ganciclovir-based genetic approach allows for much finer control of ablation-type experiments, and similar approaches can be used to specify other genetic changes in particular cell populations at specified times.

The maturation-dependent vulnerability seen in both the neuronal and glial cell populations suggests potential mechanisms for the age-dependent injury seen in TBI. The nestin-HSV-TK transgenic mouse line described here allows for selective ablation of dividing progenitors during different developmental stages. Here, we demonstrate that in younger animals, injuries that occur during the peak of myelination result in tightly regulated agedependent changes that likely influence motor recovery after injury. These data suggest that injuries such as TBI that occur at very young ages are likely to effect subsequent white matter development. This has interesting implications on restorative therapies for younger children that may need to be based more on facilitating oligodendrocyte-specific recovery as opposed to older children where this population may not be as susceptible to adverse sequelae following similar injuries.
The capability to introduce specified cell ablation provides the potential for the further characterization of the age-related changes in both the neuronal and glial progenitor populations during normal development and in response to insults such as TBI. This feature can be quite valuable in the study of the influence of age on recovery from brain injury. While TBI is devastating to young and old alike, the ability to regain at least some functions following injury, along with the increasing evidence of continued neural integration into adulthood, demonstrates that there is still substantial promise for the development of novel therapies that might one day make for more complete recovery following TBI.

\section{Acknowledgments}

This study was supported by NIH grant R01 NS048192 (S.G.K.) and the Seay Endowed Fund for Research on Brain and Spinal Cord Injuries in Children (S.G.K.) and by the Green Fellowship Program, the University of Texas at Dallas and UT Southwestern (J.W.).

\section{References}

-Altman J, Das GD (1965): Autoradiographic and histological evidence of postnatal hippocampal neurogenesis in rats. J Comp Neurol 124:319-335.

Back SA, Han BH, Luo NL, Chricton CA, Xanthoudakis S, Tam J, Arvin KL, Holtzman DM (2002): Selective vulnerability of late oligodendrocyte progenitors to hypoxia-ischemia. J Neurosci 22:455-463.

Back SA, Luo NL, Borenstein NS, Levine JM, Volpe JJ, Kinney HC (2001): Late oligodendrocyte progenitors coincide with the developmental window of vulnerability for human perinatal white matter injury. J Neurosci 21:1302-1312.

- Back SA, Riddle A, McClure MM (2007): Maturation-dependent vulnerability of perinatal white matter in premature birth. Stroke 38: 724-730.

Baumann N, Pham-Dinh D (2001): Biology of oligodendrocyte and myelin in the mammalian central nervous system. Physiol Rev 81: 871-927.

Covey MV, Jiang Y, Alli VV, Yang Z, Levison SW: Defining the critical period for neocortical neurogenesis after pediatric brain injury. Dev Neurosci 2010; DOI: 10.1159/000321607.
Emsley JG, Mitchell BD, Kempermann G, Macklis JD (2005): Adult neurogenesis and repair of the adult CNS with neural progenitors, precursors, and stem cells. Prog Neurobiol $75: 321-341$

Gage FH, Coates PW, Palmer TD, Kuhn HG, Fisher LJ, Suhonen JO, Peterson DA, Suhr ST, Ray J (1995): Survival and differentiation of adult neuronal progenitor cells transplanted to the adult brain. Proc Natl Acad Sci USA 92:11879-11883

-Gale SD, Prigatano GP (2010): Deep white matter volume loss and social reintegration after traumatic brain injury in children. J Head Trauma Rehabil 25:15-22.

Giza CC, Kolb B, Harris NG, Asarnow RF, Prins ML (2009): Hitting a moving target: basic mechanisms of recovery from acquired developmental brain injury. Dev Neurorehabil 12:255-268.

Giza CC, Prins ML (2006): Is being plastic fantastic? Mechanisms of altered plasticity after developmental traumatic brain injury. Dev Neurosci 28:364-379.

Goold D, Vane DW (2009): Evaluation of functionality after head injury in adolescents. J Trauma 67:71-74, discussion 74 .
Gourevitch R, Rocher C, le Pen G, Krebs M-O, Jay TM (2004): Working memory deficits in adult rats after prenatal disruption of neurogenesis. Behav Pharmacol 15:287-292.

He Y, Cai W, Wang L, Chen P (2009): A developmental study on the expression of PDGF $\alpha$ R immunoreactive cells in the brain of postnatal rats. Neurosci Res 65:272-279.

Jin K, Minami M, Lan JQ, Mao XO, Batteur S, Simon RP, Greenberg DA (2001): Neurogenesis in dentate subgranular zone and rostral subventricular zone after focal cerebral ischemia in the rat. Proc Natl Acad Sci USA 98: $4710-4715$.

Johnston MV (2009): Plasticity in the developing brain: implications for rehabilitation. Dev Disabil Res Rev 15:94-101.

Kernie SG, Parent JM (2010): Forebrain neurogenesis after focal ischemic and traumatic brain injury. Neurobiol Dis 37:267-274.

Koch JD, Miles DK, Gilley JA, Yang CP, Kernie SG (2008): Brief exposure to hyperoxia depletes the glial progenitor pool and impairs functional recovery after hypoxic-ischemic brain injury. J Cereb Blood Flow Metab 28: 1294-1306 
Leuner B, Gould E, Shors TJ (2006): Is there a link between adult neurogenesis and learning? Hippocampus 16:216-224.

Luskin MB (1993): Restricted proliferation and migration of postnatally generated neurons derived from the forebrain subventricular zone. Neuron 11:173-189.

-Miles DK, Kernie SG (2008): Hypoxic-ischemic brain injury activates early hippocampal stem/progenitor cells to replace vulnerable neuroblasts. Hippocampus 18:793-806.

-Ness JK, Scaduto RC Jr, Wood TL (2004): IGF-I prevents glutamate-mediated bax translocation and cytochrome $\mathrm{C}$ release in O4+ oligodendrocyte progenitors. Glia 46:183-194.

- Nishiyama A, Komitova M, Suzuki R, Zhu X (2009): Polydendrocytes (NG2 cells): multifunctional cells with lineage plasticity. Nat Rev Neurosci 10:9-22.

- Parent JM, Vexler ZS, Gong C, Derugin N, Ferriero DM (2002): Rat forebrain neurogenesis and striatal neuron replacement after focal stroke. Ann Neurol 52:802-813

- Pringle NP, Mudhar HS, Collarini EJ, Richardson WD (1992): PDGF receptors in the rat CNS: during late neurogenesis, PDGF $\alpha$-receptor expression appears to be restricted to glial cells of the oligodendrocyte lineage. Development 115:535-551.

-Richardson RM, Sun D, Bullock MR (2007) Neurogenesis after traumatic brain injury. Neurosurg Clin N Am 18:169-181, xi.
Rola R, Raber J, Rizk A, Otsuka S, Vandenberg SR, Morhardt DR, Fike JR (2004): Radiationinduced impairment of hippocampal neurogenesis is associated with cognitive deficits in young mice. Exp Neurol 188:316-330.

Rothstein RP, Levison SW (2005): Gray matter oligodendrocyte progenitors and neurons die caspase-3 mediated deaths subsequent to mild perinatal hypoxic/ischemic insults. Dev Neurosci 27:149-159.

-Sauvageot CM, Stiles CD (2002): Molecular mechanisms controlling cortical gliogenesis. Curr Opin Neurobiol 12:244-249.

Scholzen T, Gerdes J (2000): The Ki-67 protein: from the known and the unknown. J Cell Physiol 182:311-322.

-Segovia KN, McClure M, Moravec M, Luo NL, Wan Y, Gong X, Riddle A, Craig A, Struve J, Sherman LS, Back SA (2008): Arrested oligodendrocyte lineage maturation in chronic perinatal white matter injury. Ann Neurol 63:520-530.

-Shors TJ, Miesegaes G, Beylin A, Zhao M, Rydel T, Gould E (2001): Neurogenesis in the adult is involved in the formation of trace memories. Nature 410:372-376.

Sookplung P, Vavilala MS (2009): What is new in pediatric traumatic brain injury? Curr Opin Anaesthesiol 22:572-578.

Suhonen JO, Peterson DA, Ray J, Gage FH (1996): Differentiation of adult hippocampus-derived progenitors into olfactory neurons in vivo. Nature 383:624-627.

- Takashima S, Itoh M, Oka A (2009): A history of our understanding of cerebral vascular development and pathogenesis of perinatal brain damage over the past 30 years. Semin Pediatr Neurol 16:226-236.
Tasker RC (2006): Changes in white matter late after severe traumatic brain injury in childhood. Dev Neurosci 28:302-308.

- Tasker RC, Salmond CH, Westland AG, Pena A, Gillard JH, Sahakian BJ, Pickard JD (2005): Head circumference and brain and hippocampal volume after severe traumatic brain injury in childhood. Pediatr Res 58:302-308.

Volpe JJ (2003): Cerebral white matter injury of the premature infant: more common than you think. Pediatrics 112:176-180.

Winocur G, Wojtowicz JM, Sekeres M, Snyder JS, Wang S (2006): Inhibition of neurogenesis interferes with hippocampus-dependent memory function. Hippocampus 16:296304

Wojtowicz JM (2006): Irradiation as an experimental tool in studies of adult neurogenesis. Hippocampus 16:261-266.

Woodruff RH, Tekki-Kessaris N, Stiles CD, Rowitch DH, Richardson WD (2001): Oligodendrocyte development in the spinal cord and telencephalon: common themes and new perspectives. Int J Dev Neurosci 19:379385.

-Yu TS, Zhang G, Liebl DJ, Kernie SG (2008): Traumatic brain injury-induced hippocampal neurogenesis requires activation of early nestin-expressing progenitors. J Neurosci 28:12901-12912. 